\title{
Evidence of the entanglement constraint on wave-particle duality using the IBM Q quantum computer
}

\author{
Nicolas Schwaller $\odot,{ }^{1, *}$ Marc-André Dupertuis, ${ }^{1, \dagger}$ and Clément Javerzac-Galy ${ }^{1,2, \ddagger}$ \\ ${ }^{1}$ Institute of Physics, École Polytechnique Fédérale de Lausanne (EPFL), Lausanne, CH-1015, Switzerland \\ ${ }^{2}$ Miraex, EPFL Innovation Park, Bâtiment L, Lausanne, CH-1015, Switzerland
}

(Received 22 February 2020; accepted 13 January 2021; published 9 February 2021)

\begin{abstract}
We experimentally verify the link existing between entanglement and the amount of wave-particle duality in a bipartite quantum system with superconducting qubits in the IBM Q quantum computer. We consider both pure and mixed states, and study the influence of state purity on the observation of the complementarity triality relation of Jakob and Bergou [arXiv:quant-ph/0302075; Opt. Commun. 283, 827 (2010)]. This work confirms the quantitative completion of local Bohr's complementarity principle by the nonlocal quantum entanglement typical of a truly bipartite quantum system.
\end{abstract}

DOI: 10.1103/PhysRevA.103.022409

\section{INTRODUCTION}

In 1924, physicist Louis De Broglie developed the theory of electron waves [1], coming up with the idea that particles behave like waves. This discovery is without a doubt one of the most stunning ideas in physics. Indeed, four years later, Niels Bohr formulated his principle of complementarity [2], dealing with this nonintuitive property of nature. It is possible to detect particle and wave characteristics of a single quantum object, but it never behaves fully like a wave and a particle at the same time. This idea was democratized by Richard Feynman in 1965, who underlined the strangeness of the so-called wave-particle duality: "a phenomenon which is impossible, absolutely impossible, to explain in any classical way, and which has in it the heart of quantum mechanics. In reality, it contains the only mystery." [3] Experiments have been conducted with photons, electrons, neutrons, atoms and large molecules (see [4] and references therein), typically with double-slit setups, where a single quantum object has two possible paths, before reaching a screen where its position is measured. Knowing which path the object took indicates the object is a pointlike particle, whereas observing an interference pattern on the screen, formed by the detected positions of the particles when the experiment is repeated, is the manifestation of the wave characteristic of the object, which apparently passes through both slits at once.

In 1979, Wootters and Zurek initiated a quantitative approach to wave-particle duality in a double slit experiment [5], applicable to intermediate cases where the wave character would be incompletely revealed and, in 1988, their surprising

\footnotetext{
*nicolas.schwaller@epfl.ch

${ }^{\dagger}$ marc-andre.dupertuis@epfl.ch

cjg@miraex.com
}

Published by the American Physical Society under the terms of the Creative Commons Attribution 4.0 International license. Further distribution of this work must maintain attribution to the author(s) and the published article's title, journal citation, and DOI. results prompted a simpler mathematical description of Bohr's principle by Greenberger and Yasin [6], namely,

$$
V^{2}+P^{2} \leqslant 1 \text {, }
$$

where $V, P \in[0,1]$ are, respectively, the a priori fringe visibility and the which-way a priori distinguishability (also called predictability). $V$ is commonly associated to the waviness and $P$ to the particleness of a single quantum object. Equality holds for pure states, or "coherent" beams. Such type of inequality was later investigated and extended by a few authors. Jaeger et al. [7] proved a similar relationship in bipartite systems (two-particle interferometer) by relating the visibility $V$ of one-particle interference fringes to the visibility of two-particle fringes $V_{12} \in[0,1]$, i.e., $V^{2}+V_{12}^{2} \leqslant 1$. Englert [8] also obtained a similar result,

$$
V^{2}+D^{2} \leqslant 1
$$

where $D \in[0,1]$ is this time the a posteriori distinguishability (after detection, therefore also intrinsically bipartite). Equality holds when the which-path detector is similarly in a pure state. The analysis of the experiments for which relations of type (1) or (2) hold can thus be significantly different and easily induce misleading representations. Englert introduced a more prudent definition of the notion of (wave-particle) duality, i.e., "the observation of an interference pattern and the acquisition of which-way information are mutually exclusive" and emphasized also that duality might not be enforced only by the use of position-momentum uncertainty relations as in the historical Bohr-Einstein debates (opening another debate, a recent account on this subtle aspect of complementarity can be found in Xiao et al. [9]). The second duality relation (2) was experimentally tested by Dürr et al. [10] with an atom interferometer. For the first duality relation (1), earlier experiments in neutron interferometers implicitly tested it $[11,12]$. For subsequent work, Englert and Bergou [11,12] included a short review of experimental and theoretical work on this topic (as of 2000), and also put on record an erasure inequality quite similar to (2) but with different quantities outside our scope here. Very recently, Norrman et al. [13] also derived 
interesting vector-light complementarity relations (1) and (2) in the case of double pinhole vectorial interference, the relevant visibility then becomes the Stokes visibility for polarization modulation.

Another important appreciation of wave-particle duality came with the realization of delayed-choice experiments, proposed by Wheeler [14] as gedanken experiments. In such a case, the choice of the type of measurement is delayed at a later stage, which allows us to challenge the idea that the measurement configuration could dictate a priori the waviness or particleness of the quantum system, as if the system would adapt to the choice of measurement. Delayed choice experiments were carried out for single particles by Jacques et al. in 2008 [15] and for two particles by Ma et al. in 2009 [16]. Since then, a rich set of proposals and various experiments have been carried out: Wheeler's delayed-choice duality $[17,18]$, delayed-choice quantum erasure [19-21], and delayed-choice entanglement swapping [22] to mention a few.

Despite such important and extensive progress, it is quite obvious that duality relations envisaged so far, (1) and (2), are incomplete because they are inequalities, which can only bound duality. Indeed, if, for example, $V=1$ is measured, then $P=0$, or vice versa, but if $V=0$, nothing can be deduced about the range of $P$. This highlights in a striking way the incompleteness of duality relations (1) and (2). Things changed when people started to study quantitative complementarity occurring in composite systems (see Refs. [23,24] and references therein). The simplest case is a bipartite composite quantum system composed of two qubits. Jakob and Bergou $[23,25]$ have found the single missing quantity in this case, which turned out to be the entanglement with the second qubit (defined by the concurrence $\mathscr{C}$ ), so

$$
V^{2}+P^{2}+\mathscr{C}^{2}=1
$$

is a triality relation and an equality which holds for any pure state of the two qubits. Furthermore, this equation can be interpreted as a complementarity relation between (wave-particle) duality of any of the two single qubits on one hand (the first two terms) and quantum entanglement with the second qubit on the other hand (the remaining term). So, the amount of duality (or complementarity) in any of the two local subsystems determines the amount of bipartite nonlocal entanglement, the latter also being understood as a property which can exclude any of the two single-partite realities if $\mathscr{C}=1$. The beauty of this relationship resides in the fact that it relates the two most counterintuitive phenomenon of quantum physics, namely, wave-particle duality and quantum entanglement, in a single relation! Delayed-choice entanglement swapping experiments have now illustrated in a particularly bright manner such entanglement-separability duality for bipartite (and multipartite) systems [14].

More recently, Qian et al. [26] derived a triality relation which looks totally similar to the Jakob-Bergou relation (3). In fact, the underlying mathematics is identical, thereby explaining the same identity, even though the physical content is quite different as two completely classical beams are considered, including the polarization degree of freedom for both beams. The analogy between the vector description of two classical polarized beams and a two-qubit quantum system is well known [27], and obtained at the price of the introduction of a so-called position cebit together with the polarization cebit (standard Jones vector of one of the beams). The notion of classical entanglement that naturally ensues is still highly debated $[28,29]$ and is sometimes referred to as entanglement of degrees of freedom, single-particle entanglement, or selfentanglement, but it shows that quantum and classical physics do cross-fertilize again (see, e.g., Refs. [30,31] and references therein). While it led some to argue that the quantum-classical boundary was shifting [32], we stress that in fact only the domain of application of Bell-like inequalities changes.

Qian et al. also carried out a follow-up experiment in the quantum limit to verify a similar triality relation for single photons [33]. In this regard, it is necessary to point out that single photons can only test the very same classical structure of the field degrees of freedom: in a lossless linear optical system, the transformation of single photon creation operators is the same as for the classical beam amplitudes (which also tells us that for more than one input photon, other effects appear). So, Ref. [33] probes in fact the same triality relation as the classical experiment [26]. This type of point of view was already exposed by Spreeuw [27]; we quote his conclusion: "The term classical entanglement seems justified even though a single particle is, strictly speaking, a quantum system. Single-photon entanglement is what remained when we took the low-intensity limit of a classical electromagnetic wave."

Another example of the Jakob-Bergou relation involving entanglement of degrees of freedom is provided by a recent analysis of potential experiments with atom interferometers involving path and internal states of single atoms [34].

In the present paper, we offer an experimental check of the original Jakob-Bergou triality relation (3) for a genuine bipartite quantum system of two qubits, namely, the superconducting qubits of the IBM Q quantum computer [35], harnessed by the current fascinating progress in widely accessible quantum technologies.

\section{QUANTUM WAVINESS, PARTICLENESS AND ENTANGLEMENT}

Consider a general pure state of two qubits,

$$
|\psi\rangle=\alpha|00\rangle+\beta|01\rangle+\gamma|10\rangle+\delta|11\rangle,
$$

with $\alpha, \beta, \gamma, \delta \in \mathbb{C}$ satisfying the normalization:

$$
|\alpha|^{2}+|\beta|^{2}+|\gamma|^{2}+|\delta|^{2}=1 .
$$

The state (4) can be characterized by its density matrix:

$$
\rho=\left(\begin{array}{cccc}
\alpha \alpha^{*} & \alpha \beta^{*} & \alpha \gamma^{*} & \alpha \delta^{*} \\
\beta \alpha^{*} & \beta \beta^{*} & \beta \gamma^{*} & \beta \delta^{*} \\
\gamma \alpha^{*} & \gamma \beta^{*} & \gamma \gamma^{*} & \gamma \delta^{*} \\
\delta \alpha^{*} & \delta \beta^{*} & \delta \gamma^{*} & \delta \delta^{*}
\end{array}\right) .
$$

By convention, the first and second qubits will be, respectively, called qubit $A$ and qubit $B$. The corresponding reduced density matrices of subsystems $A$ and $B$ are

$$
\rho_{A}=\operatorname{Tr}_{B}(\rho)=\left(\begin{array}{ll}
\alpha \alpha^{*}+\beta \beta^{*} & \alpha \gamma^{*}+\beta \delta^{*} \\
\gamma \alpha^{*}+\delta \beta^{*} & \gamma \gamma^{*}+\delta \delta^{*}
\end{array}\right)
$$


and

$$
\rho_{B}=\operatorname{Tr}_{A}(\rho)=\left(\begin{array}{cc}
\alpha \alpha^{*}+\gamma \gamma^{*} & \alpha \beta^{*}+\gamma \delta^{*} \\
\beta \alpha^{*}+\delta \gamma^{*} & \beta \beta^{*}+\delta \delta^{*}
\end{array}\right) .
$$

Three central quantities $[23,25]$ can then be derived.

First, the concurrence, defined in the bipartite pure case by

$$
\mathscr{C}(\psi)=2|\alpha \delta-\beta \gamma| \text {. }
$$

The concurrence indicates the amount of entanglement between two quantum systems $[36,37]$ as it is a monotone of the entanglement of formation, $E_{f}$, which is a measure of entanglement based on the separability criterion: $E_{f}=0$ if and only if the density matrix can be written as a mixture of product states. Both $\mathscr{C}$ and $E_{f}$ take the value one for maximally entangled states.

Second, the coherence $\mathscr{V}_{k}$ between the two orthogonal states $|0\rangle$ and $|1\rangle$ of qubit $k$, which is therefore a quantity related to a single qubit. It is directly proportional to the norm of the off-diagonal elements of its density matrix and reads

$$
\mathscr{V}_{k}=2\left|\rho_{k_{12}}\right|, \quad k=A, B .
$$

Note that the counterpart of coherence in an interference experiment is the visibility.

Third, the predictability $\mathscr{P}_{k}$, which quantifies the knowledge of which proportion of the system $k$ is in state $|0\rangle$ or $|1\rangle$. It is defined by

$$
\mathscr{P}_{k}=\left|\rho_{k_{22}}-\rho_{k_{11}}\right|, \quad k=A, B .
$$

The predictability is analogous to the which-path information in an interference experiment.

By replacing the definitions (6)-(8) in Eqs. (9)-(11), it is easy to show that

$$
\mathscr{V}_{k}^{2}+\mathscr{P}_{k}^{2}+\mathscr{C}^{2}=\left(|\alpha|^{2}+|\beta|^{2}+|\gamma|^{2}+|\delta|^{2}\right)^{2} .
$$

One notices that the right-hand side of (12) is nothing else than the norm of the state (4) raised to the power of 4 . Thus, one can conclude that for a pure state $[23,25]$,

$$
\mathscr{V}_{k}^{2}+\mathscr{P}_{k}^{2}+\mathscr{C}^{2}=1 .
$$

Note that (13) remarkably claims that for a pure state of two qubits, the amount of entanglement strictly pilots the amount of duality of any qubit of the pair, namely, $\mathscr{V}_{k}^{2}+\mathscr{P}_{k}^{2}, k=$ $A, B$, which has the same value for both qubits. Conversely, Eq. (13) also nicely reflects the well-known fact that local unitary transformations on any of the qubits cannot change the amount of mutual entanglement.

\section{EXPERIMENT ON IBM Q}

We create a tunable state on the Bloch sphere with the simple circuit shown in Fig. 1 and use linear tomography to obtain $\mathscr{V}_{k}, \mathscr{P}_{k}$, and $\mathscr{C}$, and check the Jakob-Bergou relation (13) [or the related inequality (21)]. In terms of the $\alpha$ and $\theta$ parameters, the preparation stage creates (see Appendix A)

$$
|\psi\rangle=\cos \frac{\alpha}{2}|00\rangle+\cos \frac{\theta}{2} \sin \frac{\alpha}{2}|10\rangle+\sin \frac{\theta}{2} \sin \frac{\alpha}{2}|11\rangle .
$$

Then we perform a two-qubit state tomography, allowing us to retrieve the density matrix of the quantum state, an

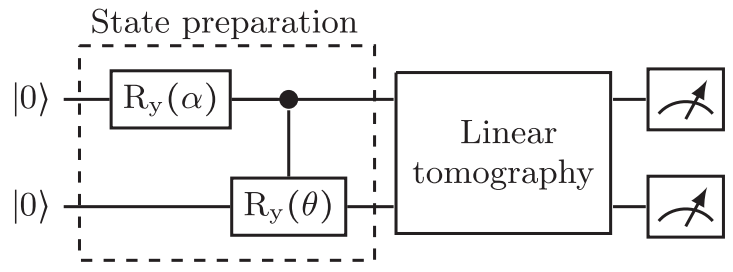

FIG. 1. Quantum circuit composed of two gates to prepare the initial state as a function of two angles, $\alpha$ and $\theta$, followed by linear tomography circuits and measurements. $\mathrm{R}_{y}$ is the usual rotation matrix, defined in appendix A.

intermediate step from which we compute $\mathscr{V}_{k}, \mathscr{P}_{k}$, and $\mathscr{C}$. The tomography procedure is the linear method proposed in Ref. [38], using the set of four Stokes measurements $\left\{\hat{\mu}_{0}=\right.$ $\left.|0\rangle\left\langle 0\left|, \hat{\mu}_{1}=\right| 1\right\rangle\left\langle 1\left|, \hat{\mu}_{2}=\right|+\right\rangle\left\langle+\left|, \hat{\mu}_{3}=\right| \circlearrowleft\right\rangle\langle\circlearrowleft|\right\}$, where $|+\rangle=$ $\frac{1}{\sqrt{2}}(|0\rangle+|1\rangle),|\circlearrowleft\rangle=\frac{1}{\sqrt{2}}(|0\rangle+i|1\rangle)$. Following linear tomography $\mathscr{V}_{k}, \mathscr{P}_{k}$, and $\mathscr{C}$ are retrieved via their direct link to the measured density matrix [Eqs. (10), (11), and (18)]. Although such a method is less direct than operational interferometric measurements of $\mathscr{V}_{k}$ and $\mathscr{P}_{k}$, it provides more accurate results since less quantum gates are required. The quantum nondemolition circuit proposed by Ref. [39] would also be necessary for an additional direct measurement of $\mathscr{C}$ (which is possible only for two-qubit states with real coefficients in the computational basis, as noted by Ref. [39]). This final measurement would also require more gates, as well as two additional ancillary qubits.

For a genuine test, it is important to check the potential falsifiability of the Jakob-Bergou relation by our measurement procedure. One may object that Eq. (13) [or the related inequality (21)] mathematically follows from the density matrix properties, hence our procedure based on tomography cannot be a serious test. This would indeed be true if one would use maximum likelyhood tomography [38], which automatically constructs perfect density matrices from a mathematical perspective, for which the Jakob-Bergou relation is always satisfied. However, in our case, we perform linear tomography which consists of the strict minimum of 16 measurements necessary to unambiguously deduce the matrix elements of a $4 \times 4$ Hermitian matrix with normalized trace, expected to be the density matrix for two qubits, but there is no safety net that would ensure automatic non-negativity of this matrix (occurrence of nonpositive matrices is well-known). Therefore, nonphysical results could also be produced for any physical observable subsequently computed with such matrices. The most likely reason is of course noise, but nonvalidity of quantum mechanics could also manifest as systematic violations, so in our case the test is genuine even if it is indirect. Most importantly, it is as strained as possible due to the limited number of gates used. Furthermore, we shall be able to check that among the results produced for $\mathscr{V}_{k}, \mathscr{P}_{k}$, and $\mathscr{C}$, there are rare violations of the Jakob-Bergou relation. Finally, we shall see that these violations disappear with longer statistical averaging, so they are clearly attributable to statistical noise, as expected.

To illustrate the equality (13), we display in Fig. 2 the values of $\mathscr{V}_{A}, \mathscr{P}_{A}$, and $\mathscr{C}$, which correspond to pure state of two qubits of the form (14), and it covers the unit sphere belonging to the first octant. We also show the position of the 


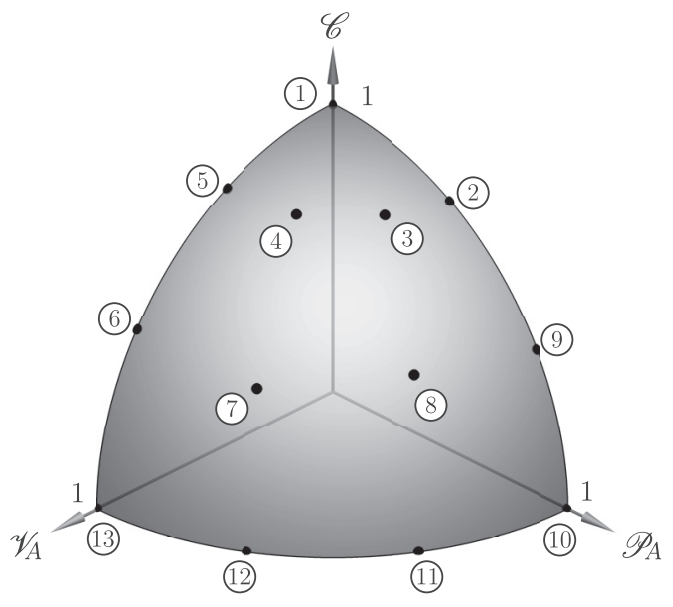

FIG. 2. Analytical $\mathscr{V}_{A}, \mathscr{P}_{A}$, and $\mathscr{C}$ for the 13 pure states described by the angles listed in Table I of Appendix A. All points lie on a sphere of unit radius.

points corresponding to the 13 states chosen in Appendix A, and which will be subsequently measured on IBMQ.

With the aim of performing the experiment on the real quantum computer, a noisy intermediate-scale quantum computer [40], formulas need to be extended to mixed states. For a mixed state with density matrix

$$
\rho=\sum_{j} p_{j}\left|\phi_{j}\right\rangle\left\langle\phi_{j}\right|
$$

where $\left|\phi_{j}\right\rangle$ are pure states composing the complete state with probabilities $p_{j}$, it is possible to compute the concurrence [41] by defining the spin flip matrix

$$
\Sigma=\sigma_{y} \otimes \sigma_{y}
$$

where $\sigma_{y}$ is the standard Pauli matrix, and the matrix

$$
R(\rho)=\rho \Sigma \rho^{*} \Sigma .
$$

The concurrence is given by

$$
\mathscr{C}=\max \left(0, \sqrt{r_{1}}-\sqrt{r_{2}}-\sqrt{r_{3}}-\sqrt{r_{4}}\right),
$$

where $r_{1} \geqslant r_{2} \geqslant r_{3} \geqslant r_{4}$ are the eigenvalues of $R(\rho)$. Using expressions (16) to (18) allows us to compute the concurrence of the pair of qubits from the linear tomography step.

The coherence of the qubit $k$ in the mixed bipartite case [42] is given by

$$
\mathscr{V}\left(\rho_{k}\right)=2\left|\operatorname{Tr}\left(\rho_{k} \sigma_{+}^{(k)}\right)\right|,
$$

where $\sigma_{+}^{(k)}=\left(\begin{array}{ll}0 & 1 \\ 0 & 0\end{array}\right)$ is the raising operator acting on qubit $k$. It can be written as

$$
\mathscr{V}_{k}=\sum_{i \neq j}\left|\rho_{k_{i j}}\right|
$$

which, for a pure state, is equivalent to (10) thanks to the Hermiticity of the density matrix. Similarly, the predictability (of the state) of a qubit [42] is given by (11) in the case of a two-qubit mixed state.

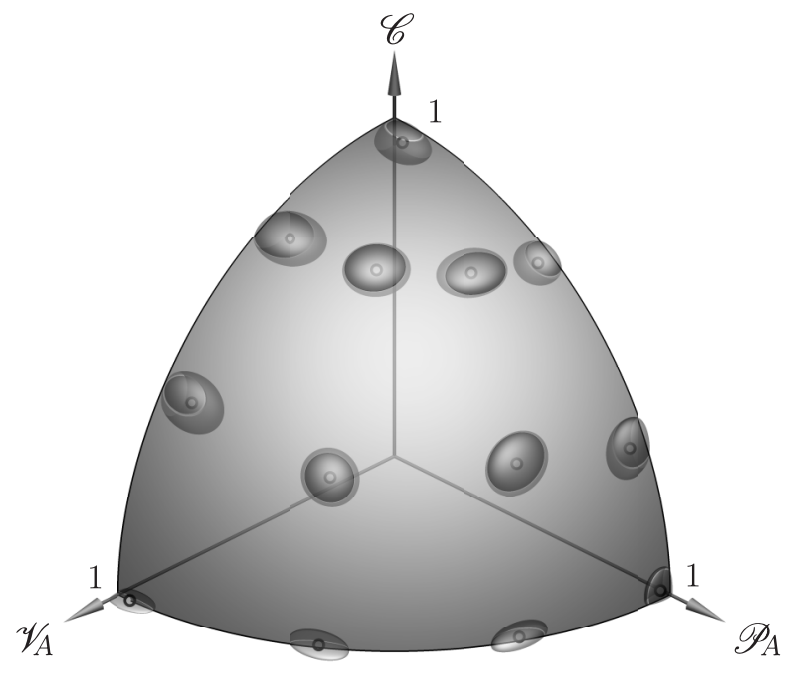

(a)

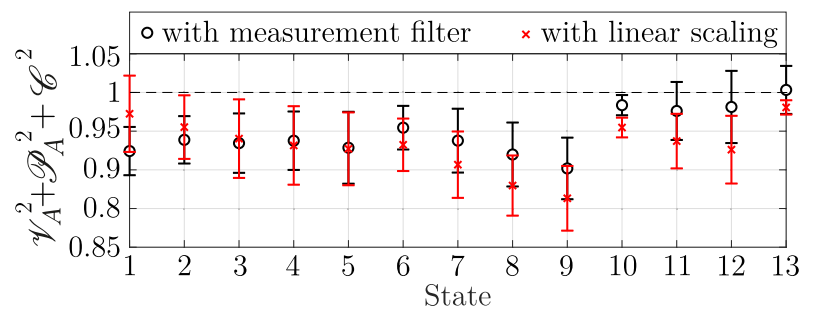

(b)

FIG. 3. (a) Evaluations of $\mathscr{V}_{A}, \mathscr{P}_{A}$, and $\mathscr{C}$ for the states introduced in Fig. 2, corrected with qiskit ignis measurement filter. For visibility ellipsoids represent $3 \sigma$ deviation from the mean. (b) Check of triality relation (21) from the same data, error bars correspond this time to standard deviation. Results with measurement filter and linear scaling of $\mathscr{C}$ can be compared.

\section{A. Experimental results}

Given the possibility to compute the quantities for a mixed state, we can now perform the experiment with the real qubits. For this, the backend ibmq_rome is used [43]. We perform 1000 shots for each of the 16 linear tomography circuits used to compute a density matrix and to subsequently evaluate $\mathscr{V}_{k}$, $\mathscr{P}_{k}$, and $\mathscr{C}$. Then each experiment is repeated 100 times to be able to evaluate the distribution of the results. Figure 3(a) shows the experimental results corresponding to Fig. 2.

In each case, $3 \sigma$-ellipsoids centered around mean values are represented and give an idea of the fitted normal distribution of $\mathscr{V}_{A}, \mathscr{P}_{A}$, and $\mathscr{C}$. In Fig. 3(a), we have used the error mitigation tool provided by qiskit ignis [44] for the two corresponding qubits. In this process, a measurement filter is computed from the outcomes of the measurements of the four computational basis states. Following the toolbox, the filter is applied to the raw measurement outcomes of each of our circuits before the density matrices are evaluated. This is supposed to compensate for the noise and decoherence taking place in the ibmq_rome backend, and we see that indeed the results agree quite well with Fig. 2. A closer look at the distance from Jakob-Bergou equality is provided by the complementary Fig. 3(b), where $\mathscr{V}_{A}^{2}+\mathscr{P}_{A}^{2}+\mathscr{C}^{2}$ is 


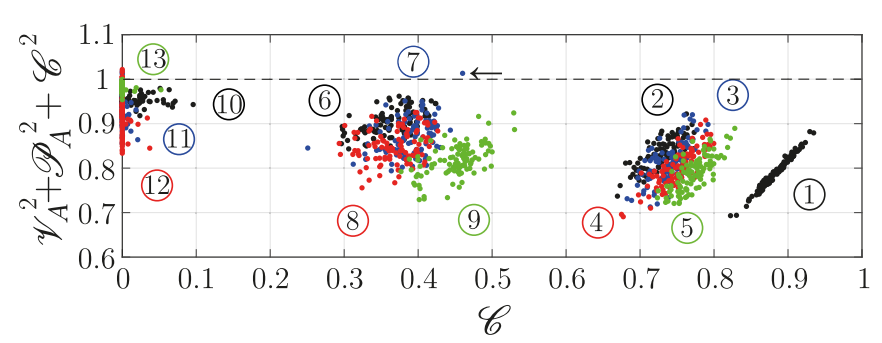

FIG. 4. Raw values [43] of $\mathscr{V}_{A}^{2}+\mathscr{P}_{A}^{2}+\mathscr{C}^{2}$ appearing in the triality relation (21) for the 13 states introduced in Fig. 2. The arrow shows a result violating the Jakob-Bergou inequality.

reported for each state. We see that error mitigation is roughly equivalent to upscaling the $\mathscr{C}$ axis of the raw results by $\sim 1 / 0.899$, while leaving $\mathscr{V}_{A}, \mathscr{P}_{A}$ unchanged. On the equator, where entanglement between the two qubits is vanishing (not the most interesting part), the equality is best satisfied. For all other states with nonvanishing concurrence, we see that $\mathscr{V}_{A}^{2}+\mathscr{P}_{A}^{2}+\mathscr{C}^{2}$ clearly falls slightly below unity (this is even more true without error mitigation or upscaling, cf. Fig. 4). This is not surprising since the mixedness of the state (due to unavoidable experimental decoherence and noise) implies [23,25]

$$
\mathscr{V}_{k}^{2}+\mathscr{P}_{k}^{2}+\mathscr{C}^{2} \leqslant 1 \quad(k=A, B) .
$$

In the next section, we shall further prove that the limited purity of the generated state does exclusively explain the maximum observed concurrence level (and justifies the scaling factor).

Figure 4 offers a closer look at the 100 measured raw values corresponding to Fig. 3(a), as a function of $\mathscr{C}$. Two features are noteworthy. First, for the states with highest concurrence (states 1-4), we see elongated clouds which show that the higher the concurrence the better the equality in (21). It is a manifestation of the fact that purity limits concurrence, as shown in the next section. The second feature worth noting is the existence of points clearly violating the Jakob-Bergou inequality (only one with $\mathscr{C}>0$, indicated by the arrow). In such a case, we checked that the intermediate density matrix is also nonpositive (necessary but nonsufficient prerequisite). The frequency of such violations does strongly diminish with the number of shots used before the intermediate density matrix evaluation (and they are already rare for 1000 shots). This disappearance is in accordance with the assumption that they are due to noise and proves that the Jakob-Bergou relation is valid, just as quantum mechanics. However, their mere existence confirms the potential falsifiability of the Jakob-Bergou relation in our experiment.

\section{B. Purity limits concurrence}

To improve and understand better the scaling observed between the experimental concurrence $\mathscr{C}_{\text {exp }}$ and its theoretical counterpart $\mathscr{C}_{\text {th }}$, we carry out a second experiment. Now a thousand states with random values of $\alpha$ and $\theta$ are generated and measured using the ibmq_rome backend [45], this time with a larger number of 8192 shots per circuit. For each of these states, the raw values of $\mathscr{V}_{k}, \mathscr{P}_{k}$, and $\mathscr{C}$ are displayed for qubits $A$ and $B$ in Appendix B (Fig. 7(a)) and no viola-

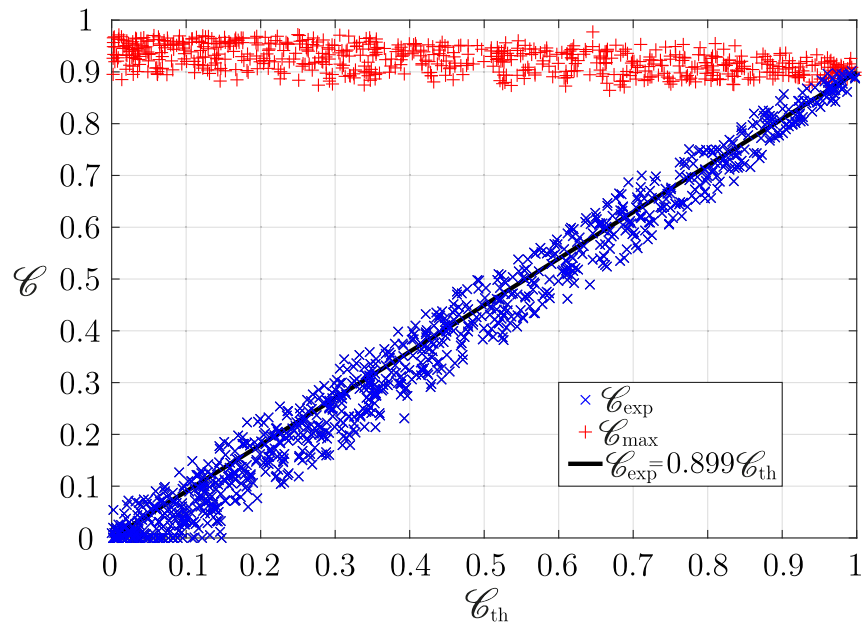

FIG. 5. Concurrence $\mathscr{C}_{\text {exp }}$ for a given theoretical $\mathscr{C}_{\text {th }}$; linear scaling used between $\mathscr{C}_{\text {exp }}$ and $\mathscr{C}_{\text {th }}$ is also shown as a straight line. $\mathscr{C}_{\text {max }}$ is computed for all positive definite density matrices.

tion of the Jakob-Bergou inequality is observed anymore. We have also measured a negative correlation (Pearson correlation coefficient of -0.418$)$ between the state purity $\operatorname{Tr}\left(\rho^{2}\right)$ and the distance $\mathscr{C}_{\text {th }}-\mathscr{C}_{\text {exp }}$. This fact is not surprising, as entanglement is a fragile resource [46] to the environment, and concurrence is known to be limited by the state purity [47].

In fact, it is possible to quantify the drop of concurrence which is due to the mixedness of the two-qubit state. Indeed, for all mixed states $\rho$ with given purity (i.e., characterized by a given set of eigenvalues $\left.\lambda_{1} \geqslant \lambda_{2} \geqslant \lambda_{3} \geqslant \lambda_{4} \geqslant 0\right)$, there is a rigorous upper bound on possible concurrence [37,48]:

$$
\mathscr{C}_{\max }(\rho)=\max \left(0, \lambda_{1}-\lambda_{3}-2 \sqrt{\lambda_{2} \lambda_{4}}\right) .
$$

For all of our random states which have positive measured density matrix, it is possible to compute $\mathscr{C}_{\max }$. Figure 5 displays $\mathscr{C}_{\text {exp }}$ and $\mathscr{C}_{\text {max }}$ as a function of the theoretical concurrence of each generated state $\mathscr{C}_{\text {th }}$. First, we see that $\mathscr{C}_{\text {exp }}$ is fairly linear as a function of $\mathscr{C}_{\text {th }}$ and that the slope is less than unity as expected. Second, and more interesting, we see that states generated on the high end of the concurrence do reach $\mathscr{C}_{\text {max }}$, showing that achievable purity is the limiting factor for concurrence, and as a result the principal cause of the flattening of the sphere along the $\mathscr{C}$ axis as observed in the raw data [Fig. 7(a)]. The linear approximation used in the previous section $\frac{\mathscr{C}_{\text {exp }}}{\mathscr{C}_{\text {th }}}=0.899$ is also displayed in Fig. 5 and corresponds to the straight line joining the two end points since they are the most relevant [and for enhanced accuracy the highest end point at maximal concurrence $(\alpha=\pi / 2, \theta=\pi)$ has been computed using a hundred density matrix evaluations].

Concurrence is an entanglement monotone, so one could say that the linearity indicates that the efficiency in the preparation of an entangled state is fairly constant, but limited on the high end by achievable purity.

\section{CONCLUSION}

Our work is an experimental explicit verification of the beautiful Jakob-Bergou triality relation for bipartite pure quantum states of two qubits [23-25]. This relation really 
represents the full quantitative completion of local Bohr's complementarity principle by quantum entanglement (concurrence) for this case. The measurements on the two superconducting qubits of the IBM Q quantum computer have shown that the duality of each qubit can indeed be turned off completely or set to any desired amount by controlling the degree of entanglement between the qubits. Clearly, the Jakob-Bergou relation can be separated into mutually exclusive local and nonlocal parts as

$$
\mathscr{S}_{k}^{2}+\mathscr{C}^{2}=1
$$

where $\mathscr{S}_{k}^{2}=\mathscr{P}_{k}^{2}+\mathscr{V}_{k}^{2}$ is the amount of locality since the predictability $\mathscr{P}_{k}$ and the visibility (coherence) $\mathscr{V}_{k}$ are local with respect to subsystem $k$. Maximal entanglement of the bipartite system $(\mathscr{C}=1)$ implies that the local realities must totally disappear $\left(\mathscr{S}_{k}=0\right)$, a synonym of the maximal amount of nonclassical nonlocal phenomena such as violations of Bell inequalities.

Finally, such experiments with the superconducting qubits of the IBM Q quantum computer could be extended in different fundamental directions. First, it would be interesting to test a generalization of the Jakob-Bergou relation derived for nonorthogonal alternatives using positive operator-valued measures (POVMs) in a similar interference and which-state information experiments for two qubits [49]:

$$
V^{2}+P^{2}+U^{2}+\mathscr{C}^{2}=1
$$

Here $\mathscr{C}$ remains the only purely bipartite quantity as before, but $V, P$ would become the nonorthogonal counterparts of visibility and predictability, and $U$ would be a new single qubit quantity involving the overlap of nonorthogonal markers. Second, the test of generalized triality relation to higher-dimensional systems like qudits (cf. Refs. [24], [50], and references therein), or even more interestingly to continuous variable systems, would also be one of the next steps (note that qudits would be emulated by collections of qubits on IBM Q). Third, we are also interested in the dynamical evolution of these relations under decoherence, which is inevitable in a quantum computer. For the resulting mixed states, it is well-known that Eqs. (3) and (24) become undersaturated inequalities (LHS $<1$ ), but more interestingly the evolution of $\mathscr{C}$ can be surprising, leading, for example, to entanglement sudden death [51], and one may wonder about the comparative evolution of each term. Fourth, one expects that multipartite quantum states which are presently realized on IBM Q quantum computers [52], and which are essential for applications in quantum information, also possess rich entanglement-separability duality relations of their own, which are of fundamental interest.

The code that supports this study is openly available in GitHub [53].

\section{ACKNOWLEDGMENTS}

We thank Dr. James R. Wootton for useful discussions and comments. We acknowledge use of the IBM Quantum Experience for this work. The views expressed are those of the authors and do not reflect the official policy or position of IBM or the IBM Quantum Experience team.
TABLE I. Couples of values $(\alpha, \theta)$ used to prepare the states of Fig. 2 with our circuit.

\begin{tabular}{lcc}
\hline \hline State & $\alpha$ & $\theta$ \\
\hline $\mathbf{1}$ & $\pi / 2$ & $\pi$ \\
$\mathbf{2}$ & 1.0472 & $\pi$ \\
$\mathbf{3}$ & 1.1230 & 3.6216 \\
$\mathbf{4}$ & 1.3181 & 4.0016 \\
$\mathbf{5}$ & $\pi / 2$ & 4.0816 \\
$\mathbf{6}$ & 0.5236 & $\pi$ \\
$\mathbf{7}$ & 0.7247 & 4.4416 \\
$\mathbf{8}$ & 1.1230 & 5.1196 \\
$\mathbf{9}$ & $\pi / 2$ & 5.1050 \\
$\mathbf{1 0}$ & 0 & 0 \\
$\mathbf{1 1}$ & 0.5236 & 0 \\
$\mathbf{1 2}$ & 1.0472 & 0 \\
$\mathbf{1 3}$ & $\pi / 2$ & 0 \\
\hline \hline
\end{tabular}

\section{APPENDIX A: STATE PREPARATION}

The left-hand side of the circuit in Fig. 1 prepares the state $|\psi\rangle=\cos \frac{\alpha}{2}|00\rangle+\cos \frac{\theta}{2} \sin \frac{\alpha}{2}|10\rangle+\sin \frac{\theta}{2} \sin \frac{\alpha}{2}|11\rangle$ by applying the unitary transformation:

$$
\begin{aligned}
& \left(\mathrm{CR}_{\mathrm{y}}(\theta)_{\mathrm{A} \rightarrow \mathrm{B}}\right)\left(\mathrm{R}_{\mathrm{y}}(\alpha) \otimes \mathbb{I}\right) \\
& =\left(\begin{array}{cccc}
1 & 0 & 0 & 0 \\
0 & 1 & 0 & 0 \\
0 & 0 & \cos \frac{\theta}{2} & -\sin \frac{\theta}{2} \\
0 & 0 & \sin \frac{\theta}{2} & \cos \frac{\theta}{2}
\end{array}\right) \\
& \times\left[\left(\begin{array}{cc}
\cos \frac{\alpha}{2} & -\sin \frac{\alpha}{2} \\
\sin \frac{\alpha}{2} & \cos \frac{\alpha}{2}
\end{array}\right) \otimes\left(\begin{array}{ll}
1 & 0 \\
0 & 1
\end{array}\right)\right] .
\end{aligned}
$$

According to Eqs. (9)-(11), such a unitary operation acting on state $|00\rangle$ allows the five quantities $\mathscr{V}_{k}, \mathscr{P}_{k}$ (with $k=A, B$ ), and $\mathscr{C}$ to reach their extremal values, i.e., 0 and 1 , as shown in Fig. 6 for $k=A$.

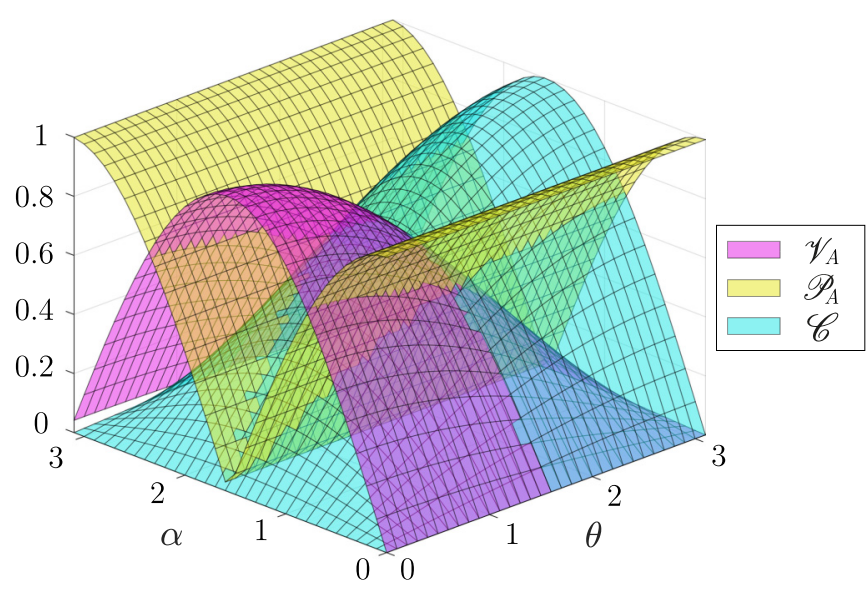

FIG. 6. Analytical values of $\mathscr{V}_{A}, \mathscr{P}_{A}$, and $\mathscr{C}$ as a function of $\alpha$ and $\theta$. 


\section{APPENDIX B: RAW VALUES IN SECOND EXPERIMENT}

Figure 7(a) reports the raw values of $\mathscr{V}_{k}$ and $\mathscr{P}_{k}(k=A, B)$, as well as $\mathscr{C}$, after 8192 shots per circuit, for 1000 two-qubit states generated with the circuit of Fig. 1 using random values of $\alpha, \theta$ uniformly sampled in $[0, \pi]$ (implying an irrelevant slight oversampling in the right corner of the octant). All points are clearly interior to the unit sphere, showing that the Jakob-Bergou triality inequality (21) is satisfied everywhere for both qubits $A, B$, and that 8192 shots are sufficient to eliminate all violations. Figure 7(b) displays in more detail the triality relation for both qubits $A, B$ as a function of concurrence.

In Fig. $7(\mathrm{c})$, we plot the purity $\operatorname{Tr}\left(\rho_{\text {exp }}^{2}\right)$ and the fidelity

$$
F=\operatorname{Tr}\left(\sqrt{\sqrt{\rho_{\mathrm{th}}} \rho_{\mathrm{exp}} \sqrt{\rho_{\mathrm{th}}}}\right)^{2}
$$

of each of the measured thousand states $\rho_{\text {exp }}$, respectively, to its target $\rho_{\text {th }}$. Not astonishingly, purity and fidelity decrease with concurrence, the latter being limited by purity (cf. Fig. 5 and its discussion in the main text).

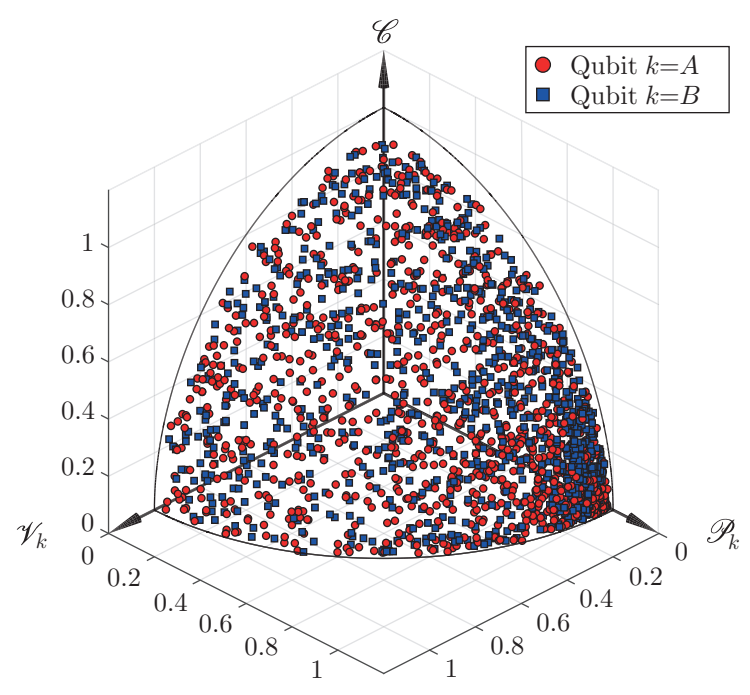

(a)

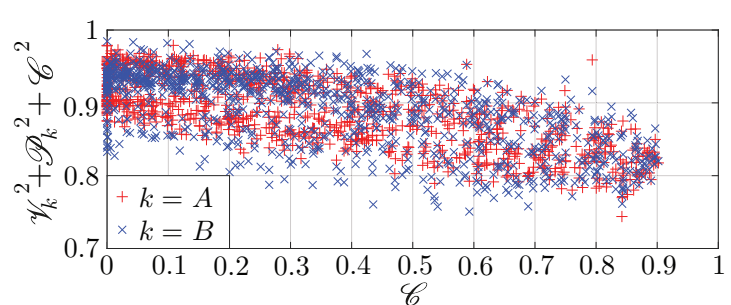

(b)

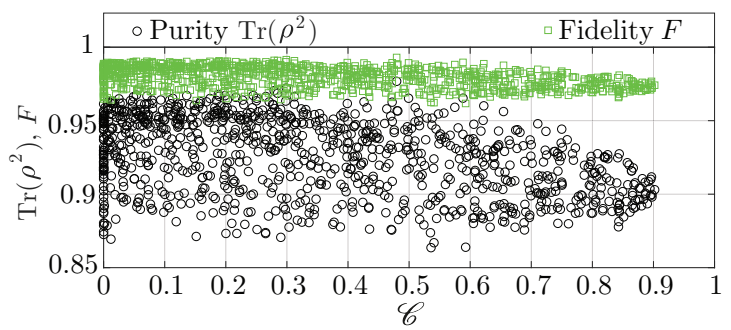

(c)

FIG. 7. Raw values for 1000 states generated with random $(\alpha, \theta)$ values [45] of (a) the triality relation for qubits $k=A, B$ (b) the triality relation for $k=A$, as a function of $\mathscr{C}$ and (c) the purity and fidelity of the two-qubit states.

[1] L. De Broglie, Research on the theory of quanta, Ph.D. thesis, Annals of Physics (Paris), 1925.

[2] N. Bohr, The quantum postulate and the recent development of atomic theory, Nature 121, 580 (1928).

[3] R. B. Bird, The Feynman lectures on physics, Richard P. Feynman, Robert B. Leighton, and Matthew Sands, Addison-Wesley, Reading, Mass, Volume I, II (1964); Volume III (1965), AIChE Journal 10, 794 (1964).

[4] O. Nairz, M. Arndt, and A. Zeilinger, Quantum interference experiments with large molecules, American J. Phys. 71, 319 (2003).

[5] W. K. Wootters and W. H. Zurek, Complementarity in the double-slit experiment: Quantum nonseparability and a quantitative statement of Bohr's principle, Phys. Rev. D 19, 473 (1979).

[6] D. M. Greenberger and A. Yasin, Simultaneous wave and particle knowledge in a neutron interferometer, Phys. Lett. A 128, 391 (1988).

[7] G. Jaeger, A. Shimony, and L. Vaidman, Two interferometric complementarities, Phys. Rev. A 51, 54 (1995).

[8] B.-G. Englert, Fringe Visibility and Which-Way Information: An Inequality, Phys. Rev. Lett. 77, 2154 (1996).
[9] Y. Xiao, H. M. Wiseman, J.-S. Xu, Y. Kedem, C.-F. $\mathrm{Li}$, and G.-C. Guo, Observing momentum disturbance in double-slit "which-way" measurements, Sci. Adv. 5, eaav9547 (2019).

[10] S. Dürr, T. Nonn, and G. Rempe, Fringe Visibility and WhichWay Information in an Atom Interferometer, Phys. Rev. Lett. 81, 5705 (1998).

[11] J. Summhammer, H. Rauch, and D. Tuppinger, Stochastic and deterministic absorption in neutron-interference experiments, Phys. Rev. A 36, 4447 (1987).

[12] B.-G. Englert and J. A. Bergou, Quantitative quantum erasure, Opt. Commun. 179, 337 (2000).

[13] A. Norrman, A. T. Friberg, and G. Leuchs, Vector-light quantum complementarity and the degree of polarization, Optica 7 , 93 (2020).

[14] X.-S. Ma, J. Kofler, and A. Zeilinger, Delayed-choice gedanken experiments and their realizations, Rev. Mod. Phys. 88, 015005 (2016).

[15] V. Jacques, E. Wu, F. Grosshans, F. Treussart, P. Grangier, A. Aspect, and J.-F. Roch, Delayed-Choice Test of Quantum Complementarity with Interfering Single Photons, Phys. Rev. Lett. 100, 220402 (2008). 
[16] X.-S. Ma, A. Qarry, J. Kofler, T. Jennewein, and A. Zeilinger, Experimental violation of a bell inequality with two different degrees of freedom of entangled particle pairs, Phys. Rev. A 79, 042101 (2009).

[17] A. R. Marlow, Mathematical Foundations of Quantum Theory (Academic Press, New York, 1978).

[18] V. Jacques, E. Wu, F. Grosshans, F. Treussart, P. Grangier, A. Aspect, and J.-F. Roch, Experimental realization of Wheeler's delayed-choice gedanken experiment, Science 315, 966 (2007).

[19] M. O. Scully and K. Drühl, Quantum eraser: A proposed photon correlation experiment concerning observation and "delayed choice" in quantum mechanics, Phys. Rev. A 25, 2208 (1982).

[20] Y.-H. Kim, R. Yu, S. P. Kulik, Y. Shih, and M. O. Scully, Delayed Choice Quantum Eraser, Phys. Rev. Lett. 84, 1 (2000).

[21] L. Mandel, Coherence and indistinguishability, Opt. Lett. 16, 1882 (1991).

[22] A. Peres, Delayed choice for entanglement swapping, J. Mod. Opt. 47, 139 (2000).

[23] M. Jakob and J. A. Bergou, Quantitative complementarity relations in bipartite systems, arXiv:quant-ph/0302075.

[24] M. Jakob and J. A. Bergou, Complementarity and entanglement in bipartite qudit systems, Phys. Rev. A 76, 052107 (2007).

[25] M. Jakob and J. Bergou, Quantitative complementarity relations in bipartite systems: Entanglement as a physical reality, Opt. Commun. 283, 827 (2010).

[26] X.-F. Qian, A. N. Vamivakas, and J. H. Eberly, Entanglement limits duality and vice versa, Optica 5, 942 (2018).

[27] R. J. C. Spreeuw, A classical analogy of entanglement, Found. Phys. 28, 361 (1998).

[28] E. Karimi and R. W. Boyd, Classical entanglement? Science 350, 1172 (2015).

[29] A. Forbes, A. Aiello, and B. Ndagano, Classically Entangled Light, in Progress in Optics (Elsevier, New York, 2019), pp. 99-153.

[30] R. J. C. Spreeuw, Classical wave-optics analogy of quantuminformation processing, Phys. Rev. A 63, 062302 (2001).

[31] T. Konrad and A. Forbes, Quantum mechanics and classical light, Contemp. Phys. 60, 1 (2019).

[32] X.-F. Qian, B. Little, J. C. Howell, and J. H. Eberly, Shifting the quantum-classical boundary: theory and experiment for statistically classical optical fields, Optica 2, 611 (2015).

[33] X.-F. Qian, K. Konthasinghe, S. K. Manikandan, D. Spiecker, A. N. Vamivakas, and J. H. Eberly, Turning off quantum duality, Phys. Rev. Research 2, 012016(R) (2020).

[34] M. Miranda and M. Orszag, Control of interference and diffraction of a three-level atom in a double-slit scheme with cavity fields, Phys. Rev. A 102, 033723 (2020).

[35] IBM Q Experience, https://quantum-computing.ibm.com.
[36] S. Hill and W. K. Wootters, Entanglement of a Pair of Quantum Bits, Phys. Rev. Lett. 78, 5022 (1997).

[37] W. K. Wootters, Entanglement of formation and concurrence, Quantum Info. Comput. 1, 27 (2001).

[38] D. F. V. James, P. G. Kwiat, W. J. Munro, and A. G. White, Measurement of qubits, Phys. Rev. A 64, 052312 (2001).

[39] F. de Melo, S. P. Walborn, J. A. Bergou, and L. Davidovich, quantum Nondemolition Circuit for Testing Bipartite Complementarity, Phys. Rev. Lett. 98, 250501 (2007).

[40] J. Preskill, Quantum computing in the NISQ era and beyond, Quantum 2, 79 (2018).

[41] W. K. Wootters, Entanglement of formation of an arbitrary state of two qubits, Phys. Rev. Lett. 80, 2245 (1998).

[42] T. E. Tessier, Complementarity relations for multi-qubit systems, Found. Phys. Lett. 18, 107 (2005).

[43] Measurements performed on ibmq_rome, November 2 to 8 , 2020, 1000 shots per circuit.

[44] H. Abraham, AduOffei, R. Agarwal, I. Y. Akhalwaya, G. Aleksandrowicz, T. Alexander, M. Amy, E. Arbel, Arijit02, A. Asfaw et al., Qiskit: An open-source framework for quantum computing (Zenodo, 2019), doi: 10.5281/zenodo.2562110.

[45] Measurements performed on ibmq_rome, November 7 to 16 , 2020, 8192 shots per circuit.

[46] R. Horodecki, P. Horodecki, M. Horodecki, and K. Horodecki, Quantum entanglement, Rev. Mod. Phys. 81, 865 (2009).

[47] M. Ziman and V. Bužek, Concurrence versus purity: Influence of local channels on Bell states of two qubits, Phys. Rev. A 72, 052325 (2005).

[48] F. Verstraete, K. Audenaert, and B. De Moor, Maximally entangled mixed states of two qubits, Phys. Rev. A 64, 012316 (2001).

[49] K. M. Fonseca-Romero, J. G. G. de Oliveira, Jr., J. G. Peixoto de Faria, and M. C. Nemes, Interference and which-state information for nonorthogonal states, Phys. Rev. A 90, 062102 (2014)

[50] W. Wu and J. Wang, Wave-particle-entanglement-ignorance complementarity for general bipartite systems, Entropy 22, 813 (2020).

[51] M. P. Almeida, F. de Melo, M. Hor-Meyll, A. Salles, S. P. Walborn, P. H. S. Ribeiro, and L. Davidovich, Environmentinduced sudden death of entanglement, Science 316, 579 (2007).

[52] D. Cruz, R. Fournier, F. Gremion, A. Jeannerot, K. Komagata, T. Tosic, J. Thiesbrummel, C. L. Chan, N. Macris, M.-A. Dupertuis, and C. Javerzac-Galy, Efficient quantum algorithms for GHZ and W States, and implementation on the IBM Quantum Computer, Adv. Quantum Technol. 2, 1970031 (2019).

[53] https://github.com/NicoSchwaller/Duality-and-Entanglementof-two-Qubits. 